\title{
Clinical Presentation of Staphylococcus epidermidis Septic Arthritis Following Anterior Cruciate Ligament Reconstruction
}

\author{
Young Mo Kim, MD, PhD and Yong Bum Joo, MD \\ Department of Orthopaedic Surgery, Chungnam National University School of Medicine, Daejeon, Korea
}

\begin{abstract}
Purpose: This study evaluated the clinical presentations of Staphylococcus epidermidis (S. epidermidis) septic arthritis after arthroscopic anterior cruciate ligament reconstruction (ACLR).

Materials and Methods: Between September 2006 and February 2008, S. epidermidis septic arthritis developed in six patients following arthroscopic ACLR. We reviewed the medical records retrospectively and interviewed the patients.

Results: The average interval between ACLR and symptom onset was 4.7 days (range, 2 to 8 days). Local warmth, swelling limited knee motion and general malaise were observed in varying degrees in all the patients. The fever was generally slight with an intermittent spiking pattern. Postoperative erythrocyte sedimentation rate and C-reactive protein were elevated. After the development of infection, the joint was aspirated in all cases. The white blood cell count was at least 22,400 , with the presence of 80 to $90 \%$ of polymorphonuclear cells. All the patients had a few Gram-positive cocci and positive cultures for $S$. epidermidis.

Conclusions: The symptoms, signs and laboratory findings of $S$. epidermidis septic arthritis following ACLR are nonspecific with various clinical presentations. So the diagnosis is often delayed. Positive gram staining can be used to confirm septic arthritis. Careful observation for septic arthritis is advised in case of presence of any abnormality after ACLR.
\end{abstract}

Key words: Knee, Septic arthritis, Staphylococcus epidermidis, Anterior cruciate ligament reconstruction.

\section{Introduction}

Arthroscopic anterior cruciate ligament reconstruction (ACLR) has become one of the most common procedures in knee surgery. Numerous complications have been reported to be associated with ACLR. Septic arthritis after ACLR has received little attention in the orthopedic literature as it is relatively uncommon

Received April 28, 2011; Revised (1st) June 26, 2011;

(2nd) August 23, 2011; Accepted August 25, 2011.

Correspondence to: Yong Bum Joo, MD.

Department of Orthopaedic Surgery, Chungnam National University Hospital, 282 Munhwa-ro, Jung-gu, Daejeon 301-721, Korea.

Tel: +82-42-220-7352, Fax: +82-42-252-7098

Email: longman76@hanmail.net

This is an Open Access article distributed under the terms of the Creative Commons Attribution Non-Commercial License (http://creativecommons.org/licenses/by-nc/3.0/) which permits unrestricted non-commercial use, distribution, and reproduction in any medium, provided the original work is properly cited. with incidence of 0.14 to $0.48 \%^{1,2)}$. Nevertheless, septic arthritis is a potentially catastrophic complication of arthroscopic ACLR and can cause problems in the joint, including the articular cartilage, and the anterior cruciate ligament (ACL) substitute. This study is a retrospective review of our experience to evaluate the clinical presentations of septic arthritis caused by Staphylococcus epidermis (S. epidermidis) after ACLR.

\section{Materials and Methods}

Between September 2006 and February 2008, a single surgeon performed 79 arthroscopic ACLRs. In all patients, a one-incision technique with two standard portals was used, and three types of grafts (four-strand hamstring autograft in 25 cases, tibialis allograft in 32 cases, and Achilles allograft in 22 cases) were employed. Just before the graft was inserted into the tibial tunnel, the prepared graft material was cultured routinely. For the femoral-side fixation of the graft material, the four-strand hamstring autograft and tibialis allograft were fixed using RigidFix (DePuy Metik, Leeds, UK), while the Achilles allograft was fixed with a metal interference screw. In all patients, an absorbable 
interference screw plus a spiked washer and screw were used for tibial-side fixation. No drain was inserted postoperatively. The rehabilitation was focused on early range of motion exercises, full passive extension, Quadriceps strength and gait training. All patients wore an ACL knee brace. Postoperative intravenous antibiotics (second generation cephalosporin) were administered for a mean of 6 days. Joint fluid was aspirated postoperatively when the patient had a feeling of discomfort, a marked effusion was noted, or any other abnormal findings, such as local warmth, were observed on the operated knee. The aspirated fluid was examined grossly, and a white blood cell (WBC) count and differential, Gram stain, and bacterial culture were performed routinely. In six patients, septic arthritis was suspected based on the development of abnormal symptoms, signs, and laboratory findings. A secondary arthroscopic operation was performed when the gross appearance and Gram stain of the final joint fluid aspirate strongly suggested an infection with persistent symptoms and signs, and an elevated C-reactive protein (CRP), irrespective of the bacterial culture results. Postoperative $S$. epidermidis septic arthritis was confirmed in all the six patients by culture. In most of the cases, culture and sensitivity test showed similar results. Especially, all the cases were sensitive to vancomycin. All the infections were eradicated successfully with one additional procedure and mean 4 weeks of intravenous antibiotic treatment was followed by 2 additional weeks of oral antibiotic treatment based on antibiotic sensitivity tests.

To evaluate the clinical features of $S$. epidermidis septic arthritis after ACLR, the charts were reviewed retrospectively and the six patients were interviewed to confirm the recorded data. We reevaluated the original procedures, the interval between the original procedure and the onset of symptoms of septic arthritis, the presenting symptoms and signs, erythrocyte sedimentation rate (ESR), CRP, number of joint fluid aspirations before and after the development of clinical symptoms of infection, the average interval between the onset of symptoms and the secondary operation, the interval between the final joint fluid aspiration and the secondary operation, the gross appearance and laboratory studies of the final joint fluid aspirate just before the secondary procedures, including the WBC count and differential, Gram stain, and culture, and the secondary procedure methods, and results of infection eradication. In addition, because we experienced an unexpectedly high rate of infection, we examined the surgical equipment and surgical team.

\section{Results}

The incidence of septic arthritis by $S$. epidermidis between September 2006 and February 2008 was $7.6 \%$. All the six patients included in this study were men, ranging in age from 20 to 53 years (mean age of 35 years) (Table 1). Two of them received a four-strand hamstring autograft, three, a tibialis allograft, and one, an Achilles allograft. Therefore, the percentage of the three kinds of grafts was $8,9.3$, and $4.5 \%$, respectively. Seven additional procedures were performed on five patients, such as partial or subtotal meniscectomy and meniscus repair. Routinely performed cultures of the prepared graft material were negative in all patients. The average interval between the original procedure and the onset of symptoms was 4.7 days (range, 2 to 8 days). In the course of septic arthritis, local warmth, joint effusion with swelling, limitation of motion, and general malaise were observed to varying degrees in all patients (Table 1). Knee pain at rest was observed in one patient only. None of the patients complained of serious symptoms until the infection reached to an advanced stage. Body temperature ranged from normal to $39.9^{\circ} \mathrm{C}$ (Fig. 1). Fever was generally slight and spiked intermittently over the development of septic arthritis, although there was continuous elevation just before the secondary operation. Considering the highest temperature each day, there was a continuous increase in temperature for several days, although the daily fever sheets showed an intermittent spiking pattern every day with periods of normal temperature during the developmental phase of septic arthritis. In addition, one patient (case 6) did not show evidence of systemic fever until the second operation was scheduled, except for the first and third postoperative days. Overall, the local and systemic symptoms and signs in the development of septic arthritis were nonspecific with various clinical presentations, and were not pronounced until a marked infection developed (Table 1). Postoperatively, the ESR and CRP were elevated to various degrees. In some patients the CRP remained elevated continuously, while in others it decreased to a relatively low level following the initial procedure and then increased rapidly (Fig. 2). In two patients (cases 2 and 6), before the development of clinical symptoms of infection, joint fluid was aspirated twice postoperatively because they had a feeling of discomfort and complained of difficulty during range of motion (ROM) exercises because of postoperative knee effusion and swelling. The aspirate was bloody, but the laboratory studies of the joint fluid were negative. In all patients, after the development of clinical symptoms of infection, joint fluid was aspirated once (cases 1 and 2) or twice (cases 3-6). In case 1, fluid was aspirated immediately 
Table 1. Data of the Six Patients Who Developed Staphylococcus epidermidis Septic Arthritis after Arthroscopic ACLR

\begin{tabular}{|c|c|c|c|c|c|c|c|c|c|c|c|}
\hline \multirow{2}{*}{$\begin{array}{c}\text { Case } \\
1\end{array}$} & \multirow{2}{*}{$\begin{array}{l}\text { Age } \\
23\end{array}$} & \multirow{2}{*}{$\begin{array}{l}\text { Sex } \\
M\end{array}$} & \multirow{2}{*}{$\begin{array}{l}\begin{array}{c}\text { Original } \\
\text { procedures }^{\text {a) }}\end{array} \\
\text { ACLR }\end{array}$} & \multirow{2}{*}{$\frac{\mathrm{Graft}^{\mathrm{b})}}{\mathrm{A}}$} & $\begin{array}{l}\text { Interval from } \\
\text { original procedure } \\
\text { to symptoms (day) }\end{array}$ & $\begin{array}{c}\text { Presenting } \\
\text { systemic/local } \\
\text { symptoms/signs }\end{array}$ & \multicolumn{2}{|c|}{$\begin{array}{c}\text { Number of } \\
\text { aspirations (before/ } \\
\text { after symptom onset) }\end{array}$} & \multicolumn{2}{|c|}{$\begin{array}{c}\text { Interval from } \\
\text { symptom onset to } \\
\text { 2nd operation (day) }\end{array}$} & \multirow{2}{*}{$\begin{array}{l}\text { Interval from final } \\
\text { aspiration to } 2 \mathrm{nd} \\
\text { operation (hr) }\end{array}$} \\
\hline & & & & & 4 & $\mathrm{~F}$ (below $38^{\circ} \mathrm{C}$ ) $/ \mathrm{M}^{2}$ & $0 / 1$ & & 12 & & \\
\hline & & & $\mathrm{LM}(\mathrm{R})$ & & & $\mathrm{H}^{1} / \mathrm{E}^{1} / \mathrm{L}^{1}$ & & & & & \\
\hline \multirow[t]{2}{*}{2} & 53 & M & $\operatorname{ACLR} M M(R)$ & $\mathrm{T}$ & 8 & $\mathrm{~F}\left(38-39^{\circ} \mathrm{C}\right) / \mathrm{M}^{2}$ & $2 / 1$ & & 5 & & 24 \\
\hline & & & LM (PM\&R) & & & $\mathrm{H}^{3} / \mathrm{E}^{1} / \mathrm{L}^{1}$ & & & & & \\
\hline \multirow[t]{2}{*}{3} & 20 & M & ACLR & $\mathrm{T}$ & 3 & $\mathrm{~F}\left(>38^{\circ} \mathrm{C}\right) / \mathrm{M}^{1}$ & $0 / 2$ & & 7 & & 36 \\
\hline & & & & & & $\mathrm{H}^{3} / \mathrm{E}^{3} / \mathrm{L}^{1}$ & & & & & \\
\hline \multirow[t]{2}{*}{4} & 33 & M & ACLR & $\mathrm{H}$ & 7 & $\mathrm{~F}\left(37.5^{\circ} \mathrm{C}\right) / \mathrm{M}^{2}$ & $0 / 2$ & & 5 & & 6 \\
\hline & & & $\mathrm{LM}(\mathrm{PM})$ & & & $\mathrm{H}^{2} / \mathrm{E}^{2} / \mathrm{L}^{1}$ & & & & & \\
\hline \multirow[t]{2}{*}{5} & 39 & M & ACLR & $\mathrm{T}$ & 2 & $\mathrm{~F}\left(38-39^{\circ} \mathrm{C}\right) / \mathrm{M}^{1}$ & $0 / 2$ & & 12 & & 24 \\
\hline & & & $\mathrm{LM}(\mathrm{PM})$ & & & $\mathrm{H}^{3} / \mathrm{E}^{2} / \mathrm{L}^{1}$ & & & & & \\
\hline \multirow[t]{2}{*}{6} & 42 & M & ACLR & $\mathrm{H}$ & 4 & No fever/ $\mathrm{M}^{1}$ & $2 / 2$ & & 11 & & 5 \\
\hline & & & $\mathrm{MM}(\mathrm{SM})$ & & & $\mathrm{P}^{2} / \mathrm{H}^{2} / \mathrm{E}^{1} / \mathrm{L}^{2}$ & & & & & \\
\hline Mean & 35 & & & & 4.7 & & & & 8.7 & & 25.7 \\
\hline Case & $\begin{array}{r}\text { Gro } \\
\text { jo }\end{array}$ & $\begin{array}{l}\text { ss apt } \\
\text { int flu }\end{array}$ & $\begin{array}{l}\text { searance of } \\
\text { iid (final) }\end{array}$ & $\begin{array}{l}\mathrm{WBC} / \mathrm{PN} \\
\text { of joint }\end{array}$ & $\begin{array}{l}\text { UN cell count } \\
\text { fluid (final) }\end{array}$ & $\begin{array}{lr}\text { ram stain of } & \text { Result } \\
\text { t fluid (final) } & \text { cult }\end{array}$ & $\begin{array}{l}\text { s of joint fluid } \\
\text { ure (final) })^{\mathrm{d})}\end{array}$ & $\begin{array}{l}\text { Final ESF } \\
(\mathrm{mm} / \mathrm{hr})\end{array}$ & $\begin{array}{c}\text { Final CRP } \\
(\mathrm{mg} / \mathrm{dL})\end{array}$ & & $\begin{array}{l}\text { Secondary } \\
\text { procedure }^{\mathrm{e})}\end{array}$ \\
\hline 1 & & ossly & purulent & 36,00 & $00 / 90 \%$ & Positive & SE & 108 & 9.95 & $\begin{array}{l}\text { Artl } \\
\text { gr }\end{array}$ & $\begin{array}{l}\text { thoscopic I\&D with } \\
\text { raft removal }\end{array}$ \\
\hline 2 & & rbid, & bloody & 22,40 & $00 / 90 \%$ & Positive & SE & 63 & 5.69 & Artl & thoscopic I\&D only \\
\hline 3 & & rbid, & bloody & 102,00 & $00 / 90 \%$ & Positive & SE & 83 & 12.71 & Artl & thoscopic I\&D only \\
\hline 4 & & rbid, & bloody & 24,00 & $00 / 80 \%$ & Positive & SE & 46 & 10.14 & Artl & thoscopic I\&D only \\
\hline 5 & & rbid, & bloody & Mar & ny/90\% & Positive & SE & 81 & 14.7 & Artl & thoscopic I\&D only \\
\hline 6 & & rbid, & bloody & 70,00 & $00 / 80 \%$ & Positive & SE & 29 & 7.1 & Artl & thoscopic I\&D only \\
\hline Mean & & & & & & & & 68.3 & 10.05 & & \\
\hline
\end{tabular}

a) ACLR: arthroscopic anterior cruciate ligament rconstruction, MM: medial meniscus, LM: lateral meniscus, R: repair, PM: partial meniscectomy, SM: subtotal meniscectomy, ${ }^{\text {b) }} \mathrm{A}$ : achilles allograft, T: tibialis allograft, $\mathrm{H}$ : four-strand hamstring autograft, ${ }^{\mathrm{c}} \mathrm{F}$ : fever, M: malaise, P: knee pain at rest, H: knee feels hot, E: effusion with swelling, L: limited knee motion, 1: mild degree, 2: moderate degree, 3: severe degree, ${ }^{\text {d) }}$ SE: Staphylococcus epidermidis, ${ }^{\mathrm{e})} \mathrm{I} \& \mathrm{D}$ : irrigation and debridement.

before the secondary operation, and it was grossly purulent with positive results for Gram staining and bacterial culture. In case 2, the joint fluid aspirated after symptom development was turbid and bloody and positive for Gram staining and bacterial culture. In cases 3-6, the initial joint aspirate after symptom development was bloody, but not turbid, and it was negative for Gram staining and bacterial culture, while a second aspirate obtained just before the secondary operation was turbid and bloody and positive for Gram staining and bacterial culture. In all patients, $S$. epidermidis was identified in the final joint aspirate, as reported after the secondary operation. The WBC count and differential of final aspirate was at least 22,400 with the presence of $80-90 \%$ polymorphonuclear cells. The average interval between symptom onset and the secondary operation was 8.7 days (range, 5 to 12 days). The average interval between the final joint aspiration and the secondary operation was 25.7 hours (range, 5 to 59 hours). The infection was eradicated after a single secondary procedure in all patients. Five patients underwent subsequent arthroscopic irrigation and debridement of the knee joint with careful inspection of the ACL graft and gentle debridement. In case 1, the graft was removed because of loss of tension and gross infection; a revision ACLR with an Achilles allograft was done 6 months later.

We could not find any source of contamination despite intensive surveillance of the surgical equipment and surgical team in an attempt to identify the origin of the infections. 


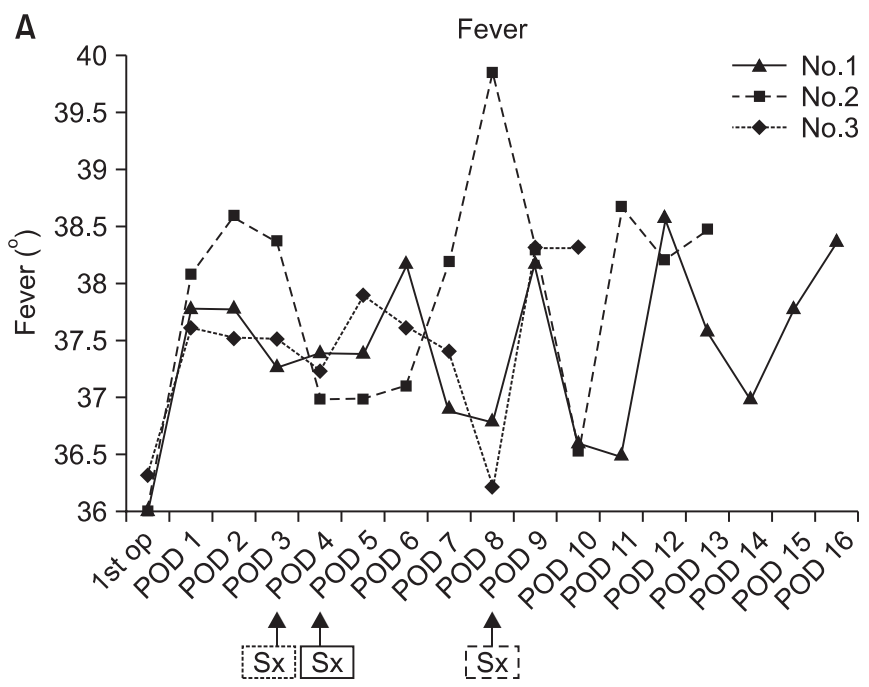

The end point of the each line is the 2 nd operation day

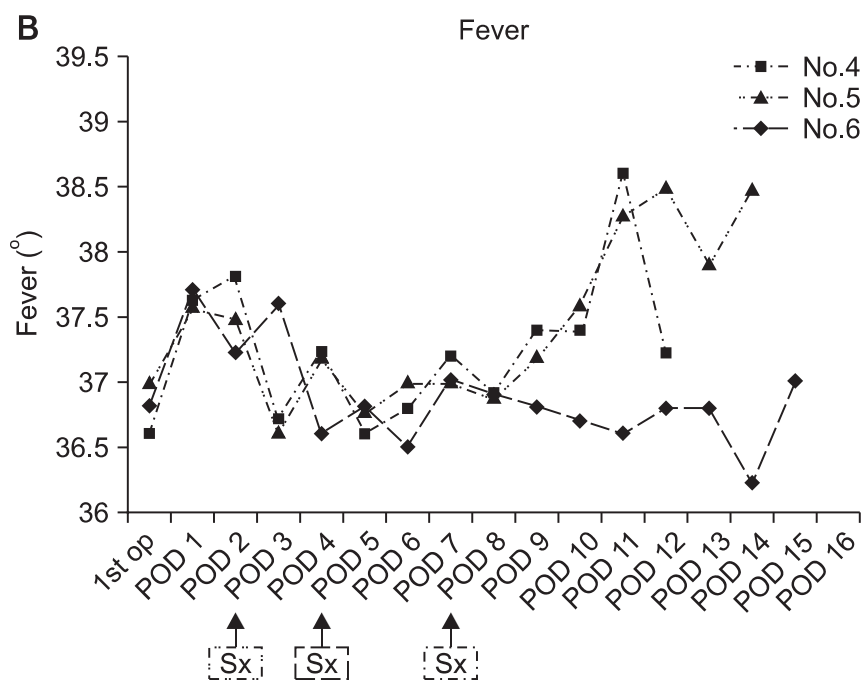

The end point of the each line is the 2 nd operation day

Fig. 1. Systemic temperature ranged from normal to $39.9^{\circ} \mathrm{C}$. Fever was generally slight and spiked intermittently in the course of development of septic arthritis, becoming elevated continuously just before the secondary operation. In one patient (case 6), no fever was seen until the second operation had been planned, except on the first and third postoperative days. The average interval between the onset of symptoms and the original procedure was 4.7 days. POD: postoperative day.

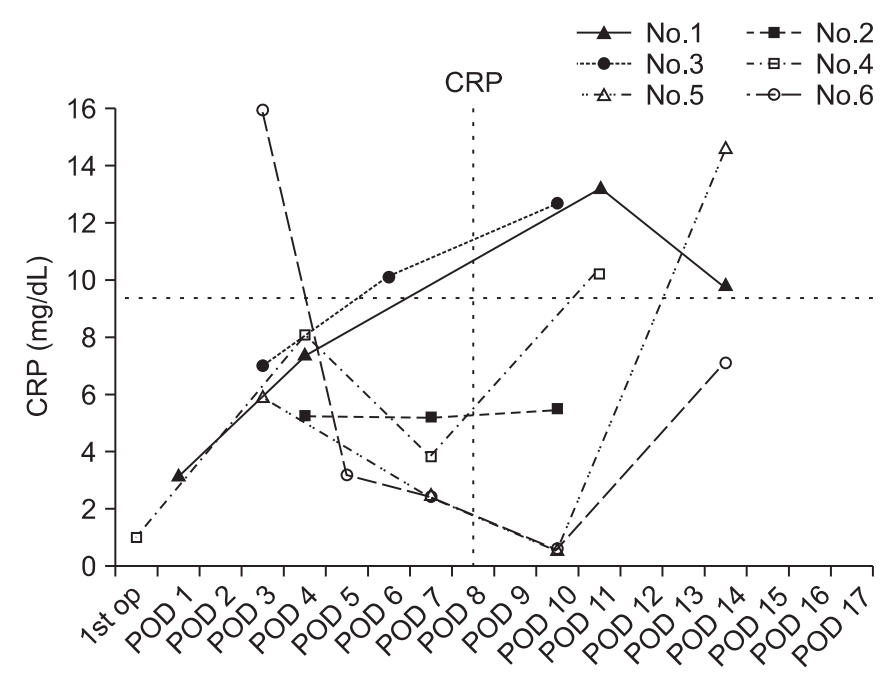

Fig. 2. Patterns of C-reactive protein (CRP) elevation. The CRP was either elevated continuously to varying degrees (cases 1-3) or elevated rapidly after an initial decrease in the postoperative peak to a relatively lower level (cases 4-6). POD: postoperative day.

\section{Discussion}

The currently reported incidence of septic arthritis following arthroscopic procedures is between 0.01 and $0.23 \%$, and the reported incidence after ACLR is between $0.14 \%$ and $0.48 \%^{1,2}$. Staphylococcus aureus (S. aureus) is the most common infecting organism and $S$. epidermidis is another frequent cause of septic arthritis after ACLR ${ }^{1,3-7)}$. Between September 2003 and February
2005, we encountered six patients (7.6\%) who developed septic arthritis after ACLR, and the causative organism was S. epidermidis. Due to the unexpectedly high incidence, we conducted intensive surveillance of the surgical equipment and surgical team because several reports have documented contamination of graft boards ${ }^{5}$, , prepackaged and sterilized sets of inflow cannulae ${ }^{6}$, and meniscus repair cannulae ${ }^{8)}$. Nevertheless, we were unable to identify the origin of the contamination. Since S. epidermidis is ubiquitous and may occasionally cause infection as a result of external contamination ${ }^{4}$, we could not rule out this possibility.

McAllister et al. ${ }^{2)}$ reported that the type of autogenous ACL graft was not a significant risk factor for septic arthritis after ACLR, and Indelli et al. ${ }^{1)}$ found no statistical difference in postoperative infection rates between allografts and autografts. They reported that allografts and autografts have the same infection risk: both respond as foreign bodies and blood supply is initially absent. Mariani and Margheritini ${ }^{4)}$ also stated that any type of graft used in ligament reconstruction of nonviable tissue could harbor bacteria. In our six patients, hamstring tendon autograft, tibialis allograft, and Achilles allograft were used.

Since septic arthritis can lead to rapid joint destruction and more than half of the glycosaminoglycan and collagen is lost within 7 days of the onset of infection ${ }^{1,7)}$, early treatment within a few days of the onset of symptoms is imperative. The classic clinical evidence of septic arthritis is swelling, rapidly progressive 
knee pain, restricted motion, local erythema, local warmth, and fever ${ }^{2,45}$. Several reports on development of septic arthritis after ACLR have stated that the clinical symptoms in all patients are similar ${ }^{2,7)}$. In our patients, the average interval between the onset of symptoms and the original procedure was 4.7 days. However, the symptoms seen during the development of septic arthritis were nonspecific with various clinical presentations, as the patients did not complain of their symptoms seriously until the advancement of infection. Local heat, joint effusion with swelling, and limitation of motion were observed in all patients, but the degree varied from mild to severe. Knee pain at rest was observed in only one patient. Armstrong et al. ${ }^{9)}$ stated that the typical postoperative pain usually lasts for only 1 or 2 days, and Williams et al. ${ }^{7)}$ considered pain, warmth, and swelling beyond this period to be signs of possible septic arthritis or soft tissue infection. As a result, we overlooked the presenting symptoms in the early stage of the development of $S$. epidermidis septic arthritis, although it has been known that $S$. epidermidis septic arthritis can produce less marked clinical symptoms, and the early clinical symptoms of infection can easily be interpreted as the ordinary postoperative status or signs of overuse inflammation ${ }^{10)}$. In addition, $S$. epidermidis septic arthritis can frequently remain as undiagnosed $^{5}$ or, diagnosed insidiously ${ }^{4}$.

A recent multicenter study ranked the infecting organism and systemic signs, such as fever and malaise, as the most important factors in the initial treatment of a postoperative infection ${ }^{11}$. In our patients, systemic fever was generally slight and showed an intermittent spiking pattern in the developmental course of septic arthritis. Moreover, in one patient (case 6), meaningful systemic fever had not been observed until the second operation was planned. All the patients experienced mild or moderate malaise.

CRP and ESR are useful routine laboratory screening markers for diagnosing potential infection. The combination of normal ESR and CRP levels reliably predicts the absence of infection, but there is a lack of reported information regarding CRP and ESR behavior following arthroscopic procedures ${ }^{12)}$. A recent multicenter study reported that the ESR was the least important factor in determining the initial treatment of postoperative infection ${ }^{11}$. Margheritini et al. ${ }^{12}$ reported that both CRP and ESR increased markedly postoperatively, peaking between the third and seventh postoperative days and subsequently decreasing after a noncomplicated arthroscopic ACLR. In addition, they reported that CRP is a more accurate predictor of postoperative complications than ESR if the blood level remains elevated or rises unexpectedly. However, such an increase in ESR and CRP might obscure the diagnosis of an early-onset infection ${ }^{5,12)}$. In our patients, the CRP exhibited two different patterns: it either remained elevated continuously (cases 1-3) or decreased after the early postoperative peak and then increased markedly just before the secondary operation (case 4-6).

Usually the leukocyte count in septic joint fluid exceeds 50,000 with predominance of neutrophils ${ }^{4}$. Cooper et al. ${ }^{3)}$ reported high WBC counts (range, 80,000-130,000) in $S$. aureus septic arthritis after ACLR. Conversely, McAllister et al. ${ }^{2)}$ and Williams et al. ${ }^{7)}$ reported that the leukocyte count in septic joint fluid was below 50,000 in some cases of septic arthritis caused by $S$. aureus, $S$. epidermidis, and Peptostreptococcus after ACLR. In our cases, the WBC count was at least 22,400 and the differential was 80 $90 \%$ polymorphonuclear cells. We thought that the leukocyte count of the joint fluid in the low-grade $S$. epidermidis septic arthritis after ACLR was less important than the gross appearance of the joint fluid and Gram stain for deciding to perform a secondary operation.

The definitive diagnosis of septic arthritis is based on the gross examination of synovial fluid, Gram stain, and culture ${ }^{1,2,4)}$. Most of the reports have described positive cultures in $100 \%$ of the cases $^{2,3,5,7)}$, although some reports have stated fewer positive results $^{6}$. In our patients, the gross appearance of final joint aspirate immediately before the secondary operation was turbid and bloody or grossly purulent, and the patients were developing symptoms and signs of infection, and had an elevated CRP. In addition, in all patients, the aspirate showed some Gram-positive cocci and cultures were positive for $S$. epidermidis. Nevertheless, the gross appearance, Gram stain, and bacterial culture of earlier joint aspirate in the development of septic arthritis were negative (except in case 1). In our cases, the presenting symptoms, signs, and laboratory findings (ESR, CRP) in the course of the development of $S$. epidermidis septic arthritis made us hesitant to perform a secondary operation. The gross appearance and Gram stain of the final aspirate influenced the decision to perform secondary arthroscopic surgery. The subsequent positive culture for $S$. epidermidis led to the selection of appropriate antibiotics after the secondary operation.

Septic arthritis in any joint requires early diagnosis and prompt decompression to minimize the severity of the sequelae. McAllister et al. ${ }^{2)}$ reported four cases of $S$. aureus septic arthritis. The infection was diagnosed within 24 hours of symptom onset, and the grafts were preserved, although the four patients required an average of 2.75 procedures after the diagnosis to eradicate the infection. Indelli et al. ${ }^{1)}$ reported six cases of septic arthritis with S. aureus infection in three, $S$. epidermidis in two, and nonhemolytic Streptococcus in one. The average interval from the 
onset of symptoms to the initial arthroscopic intervention was 7.5 days (range, 2 to 20 days). An average of 1.3 (range, 1 to 3 ) additional arthroscopic interventions was performed on five of the six patients. The graft was removed from two patients with $S$. aureus infection in whom the secondary arthroscopic surgery was performed 7 and 20 days after the onset of infection, respectively. In contrast, in the two patients who had S. epidermidis infection and underwent diagnostic arthroscopy 7 days after the onset of infection, the graft was preserved and excellent results were obtained. In our six $S$. epidermidis infections, the average interval between symptom onset and secondary arthroscopic surgery was 8.7 days (range, 5 to 12 days). The average interval between the final joint aspiration and the secondary operation was 25.7 hours (range, 5 to 59 hours). In two patients (cases 1 and 5), the secondary operation was performed 12 days after symptom onset: graft was removed in the patient (case 1) who underwent secondary arthroscopic operation 59 hours after the final aspiration and preserved in the patient (case 6) who underwent secondary arthroscopic operation 24 hours after the final aspiration. The goals of the treatment for septic arthritis after ACLR are first to protect the articular cartilage and second, to protect the graft. We postulate that to attain these purposes, prompt secondary arthroscopic operation should be performed on suspicion of septic arthritis to preserve the graft in S. epidermidis infection after arthroscopic ACLR. Arthroscopic lavage using normal saline and debridement for acute septic arthritis of the knee is a well-described and effective therapeutic intervention ${ }^{1)}$. We also treated $S$. epidermidis infection successfully with arthroscopic lavage and debridement, and only one additional operation was needed in our six patients. The limitation of our study include that we could neither confirm the cause of $S$. epidermidis septic arthritis nor rule out the possibility of contamination.

\section{Conclusions}

In $S$. epidermidis septic arthritis after ACLR, the symptoms, signs, and laboratory findings in the development of septic arthritis were nonspecific with various clinical presentations, and it was difficult to make a diagnosis and decision of performing a second operation based on them. The diagnosis tended to be delayed until the appearance of gross infection of the knee and the joint aspirate showed Gram-positive organisms. Therefore, the patient should be observed closely if any abnormalities are found after ACLR. Despite the delayed diagnosis, the graft was preserved in five of the six cases.

\section{References}

1. Indelli PF, Dillingham M, Fanton G, Schurman DJ. Septic arthritis in postoperative anterior cruciate ligament reconstruction. Clin Orthop Relat Res. 2002;(398):182-8.

2. McAllister DR, Parker RD, Cooper AE, Recht MP, Abate J. Outcomes of postoperative septic arthritis after anterior cruciate ligament reconstruction. Am J Sports Med. 1999;27:562-70.

3. Cooper A, Parker RD, Dickerson A, Maschke S. Postoperative septic arthritis in anterior cruciate ligament reconstruction. Arthroscopy. 1996;12:372.

4. Mariani PP, Marqheritini F. Septic arthritis of the knee. Tech Knee Surg. 2003;2:117-24.

5. Schollin-Borg M, Michaelsson K, Rahme H. Presentation, outcome, and cause of septic arthritis after anterior cruciate ligament reconstruction: a case control study. Arthroscopy. 2003;19:941-7.

6. Viola R, Marzano N, Vianello R. An unusual epidemic of Staphylococcus-negative infections involving anterior cruciate ligament reconstruction with salvage of the graft and function. Arthroscopy. 2000;16:173-7.

7. Williams RJ 3rd, Laurencin CT, Warren RF, Speciale AC, Brause BD, O'Brien S. Septic arthritis after arthroscopic anterior cruciate ligament reconstruction. Diagnosis and management. Am J Sports Med. 1997;25:261-7.

8. Blevins FT, Salgado J, Wascher DC, Koster F. Septic arthritis following arthroscopic meniscus repair: a cluster of three cases. Arthroscopy. 1999;15:35-40.

9. Armstrong RW, Bolding F, Joseph R. Septic arthritis following arthroscopy: clinical syndromes and analysis of risk factors. Arthroscopy. 1992;8:213-23.

10. Johnson MW. Acute knee effusions: a systematic approach to diagnosis. Am Fam Physician. 2000;61:2391-400.

11. Matava MJ, Evans TA, Wright RW, Shively RA. Septic arthritis of the knee following anterior cruciate ligament reconstruction: results of a survey of sports medicine fellowship directors. Arthroscopy. 1998;14:717-25.

12. Margheritini F, Camillieri G, Mancini L, Mariani PP. C-reactive protein and erythrocyte sedimentation rate changes following arthroscopically assisted anterior cruciate ligament reconstruction. Knee Surg Sports Traumatol Arthrosc. 2001;9:343-5. 